\title{
Interaction of an Antenna With a Hot Plasma and the Theory of Resonance Probes'
}

\author{
J. A. Fejer \\ Contribution From the Southwest Center for Advanced Studies, Post Office Box 8478, Dallas, Texas
}

(Received May 14, 1964; revised June 26, 1964)

\begin{abstract}
The impedance and the radiation field in the surrounding hot plasma are calculated for an electrically short antenna that consists of two spherical conductors excited through thin wires in phase opposition. In the calculation the pressure tensor is replaced by a scalar pressure. A discontinuous model of the ion sheath is used.

The losses due to the radiation of electromagnetic and electrostatic waves are calculated and are expressed in terms of equivalent series resistances. The operation of resonance probes is discussed. It is shown that their resonant frequency is well below the electron plasma frequency if the probe radius is much larger than the Debye length. The significance of this result to both past and future ionospheric rocket probe experiments is pointed out. The limitations of the present treatment are discussed.
\end{abstract}

\section{Introduction}

Antenna impedance measurements in the ionosphere during rocket flights show the presence of a much larger resistive component [Whale, 1963] than is predicted by the type of antenna theory in which the surrounding plasma is treated as cold. In principle the interaction of an antenna with a hot and very tenuous plasma should be treated by solving a combination of the collisionless Boltzmann equation with Maxwell's equations. The mathematical difficulties of solving these equations and satisfying the appropriate boundary conditions are, however, formidable, and therefore the problem is considerably simplified in this paper by the use of certain approximations. The first of these is the use of a scalar pressure [Spitzer, 1962, p. 24] and the resulting: neglect of Landau damping [Landau, 1946]. The problem is further simplified by the rather crude additional assumption that the plasma surrounding the antenna is sharply bounded and that outside the boundary the plasma is uniform. The effects of the earth's magnetic field and dissipative effects are neglected. For other applications of the same type of approximations the reader is referred to papers by Gould [1959], Fejer [1964], and Nickel, Parker, and Gould [1963].

These approximations are applied here to a simple dipole antenna that consists of two spheres excited in phase opposition through wires whose capacity is neglected. Two types of waves can be excited by such a dipole antenna in the uniform plasma: electromagnetic waves and acoustic-type, longitudinal electrostatic waves. The distance between the centers of the two spheres is assumed to be much shorter than the wavelength of electromagnetic waves but much longer than the wavelength of elec-

1 Presented at the Conference on Nonlinear Processes in the Ionosphere, Dec. 16, 17, 1963, Boulder, Colo. trostatic waves and than the diameter of the spheres. The term "electrostatic waves" is used in this paper to describe the plasma oscillations discussed by Tonks and Langmuir [1929] and further analyzed by Landau [1946].

The present analysis and its conclusions differ from those of Whale [1963], who also considered the excitation of electrostatic waves by introducing the concept of an isotropic pressure. Whale assumed the existence of interaction between the fluctuating quasi-electrostatic field (the near field of the electromagnetic waves) and the electrostatic waves throughout the uniform plasma. In the present treatment the only interaction is taken to occur at the inner boundary (in reality in the sheath). Within the uniform plasma, in the absence of a magnetic field, the electrostatic wave and the electromagnetic wave (or in the present limit the quasi-electrostatic field) propagate independently and therefore cannot interact in terms of the hydrodynamic approximation.

Since the completion of the present work a number of closely related papers [Cohen, 1961; Cohen, 1962a; Cohen, 1962b; Hessel and Shmoys, 1962; Chen, 1963; Harp, 1963; Pavkovich, 1964; Wait, 1964] came to the author's attention. Of these the work of Cohen, Hessel, and Shmoys, and Chen are restricted to the calculation of the electromagnetic and electrostatic wave fields of point or line current sources in hot uniform plasmas. Wait considers a finite slotted-sphere antenna immersed in a hot plasma and his work is more closely related to the present one. Pavkovich, in contrast to all other workers, does not restrict himself to the concept of an isotropic pressure and gives numerical results of a calculation based on the collisionless Boltzmann equation, for the radiofrequency properties of a one dimensional plasma sheath. His results were modified approximately for spherical geometry by Harp. This modification is closely related to the present 
work and reaches similar conclusions about the theory of the resonant probe used by Miyazaki et al. [1960]. The relationship between the present work and the work of others will be further clarified in the body of the paper.

\section{Excitation of Electrostatic Waves}

The antenna described in the introduction radiates both electrostatic and electromagnetic waves. In the present section only one of the two spheres of the antenna will be considered at first and the near field of the electromagnetic wave field will be approximated by the quasi-electrostatic field calculated from Poisson's equation. The impedance of the whole antenna, excluding the electromagnetic radiation resistance, is then simply taken to be equal to twice the impedance of a single sphere.

The quasi-electrostatic field calculated in this section for a distance large compared to the distance between the spheres but small compared to the electromagnetic wavelength will be extrapolated in the following section to much greater distances. In this manner the field of the electromagnetic wave and the electromagnetic radiation resistance of the antenna, neglected in this section, are calculated in the following section.

Let $R$ be the radius of one of the spheres and $r$ the distance of an arbitrary point from the center of the sphere. It is assumed that the unperturbed electron concentration vanishes for $r<R$, and has the constant value $N$ for $r>R$. This is admittedly a rather artificial model of the unperturbed ion sheath. It effectively assumes an abrupt potential barrier (this could be visualized as a hypothetical double layer formed from infinitely heavy positive and negative ions) at $r=R$ which prevents the penetration of electrons inside the sphere at $r=R$. It is clear that the radial component of the mean electron velocity must vanish at $r=R$. Immediately inside the sheath there is assumed to be a spherical conductor whose (quasi-electrostatic) perturbation potential is taken to be a harmonic function of the time. (In principle the radius of the conductor could be taken as smaller than the radius of the spherical plasma-free cavity without essentially modifying the analysis; this will not be done here.) Since there is no mean radial motion of the electrons at $r=R$, there is no fluctuating charged surface layer there and therefore both the perturbation potential $V$ and its normal derivative must be continuous at $r=R$. Inside the plasma the equation

$$
m N \partial \mathbf{v} / \partial t=N e \nabla V-\gamma K T \nabla n
$$

is taken to be valid where $m$ is the mass, $e$ is the absolute value of the charge, $N$ is the unperturbed number density and $n$ is the perturbation in the number density of electrons (factors $e^{i \omega t}$ are taken for granted in $n$ and in $V$ ), $K$ is Boltzmann's constant, $T$ is the temperature, and where the ratio $\gamma$ of the specific heats is taken as 3 [Spitzer, 1962]. A combination of the divergence of (1) with the equation of continuity (satisfied by the velocity $\mathbf{v}$ of the electron gas)

leads to

$$
\boldsymbol{\nabla} \cdot(N \mathbf{v})=-\partial n_{0} / \partial t
$$

$$
\frac{\partial^{2} n}{\partial t^{2}}+\frac{N e}{m} \nabla^{2} V-\frac{\gamma K T}{m} \nabla^{2} n_{t}=0 .
$$

A combination of (3) and Poisson's equation in spherical coordinates and with spherical symmetry,

$$
\nabla^{2} V=\frac{\partial^{2} V}{\partial r^{2}}+\frac{2}{r} \frac{\partial V}{\partial r}=\frac{e}{\epsilon_{0}} n
$$

leads to

$$
\nabla^{2} n=\frac{\partial^{2} n}{\partial r^{2}}+\frac{2}{r} \frac{\partial n}{\partial r}=\alpha^{2} n
$$

where $\alpha^{2}=\left(\omega_{N}{ }^{2}-\omega^{2}\right) / u^{2}, \omega_{N}=\left(e^{2} N / \epsilon_{0} m\right)^{1 / 2}$ is the electron plasma frequency and $u=(\gamma K T / m)^{1 / 2}$ is the velocity of electrostatic waves in the high frequency $\operatorname{limit}\left(\omega>>\omega_{N}\right)$.

The general solution, which vanishes at infinity, of the differential equations (4) and (5) has the form

$$
\begin{gathered}
n=\frac{C_{1}}{r} e^{-\alpha r} \\
V=\frac{e}{\epsilon_{0} \alpha^{2}} n+\frac{C_{2}}{r}
\end{gathered}
$$

where that part of the solution which is associated with the constant $C_{1}$, describes the electrostatic wave, whereas the part associated with $C_{2}$ describes a quasi-electrostatic field which is a good approximation to the electromagnetic wave field within distances very much shorter than a wavelength from the antenna.

The boundary condition $v=0$ at $r=R$ yields with the aid of (1)

$$
\left(\omega_{N}^{2} \frac{\partial V}{\partial r}\right)_{r=R}=\left(\frac{e u^{2}}{\epsilon_{0}} \frac{\partial n_{0}}{\partial r}\right)_{r=R} .
$$

Substitution of $n$ and $V$ from (6) and (7) into (8) leads to the relation

$$
C_{2}=-\frac{e}{\epsilon_{0}} \frac{u^{2} \omega^{2}}{\omega_{N}^{2}\left(\omega_{N}^{2}-\omega^{2}\right)}(1+\alpha R) e^{-\alpha R} C_{1} .
$$

Substitution of $C_{2}$ from (9) into (6) and (7) leads to the following relationship between the perturbation in the number density and the perturbation potential $V$ at $r=R$ :

$$
\frac{n(R)}{V(R)}=\frac{\epsilon_{0} \omega_{N}^{2}}{e u^{2}}\left(1-\frac{R \omega^{2}}{u\left(\omega_{N}^{2}-\omega^{2}\right)^{1 / 2}}\right)^{-1} .
$$

Similarly, the effective, complex capacity of the conductor, defined here as the ratio of the charge on the conductor to the potential $V(R)$ is given by

$$
\begin{aligned}
& C_{\text {eff }}=-\frac{4 \pi R^{2} \epsilon_{0}(\partial V / \partial r)_{r=R}}{V(R)} \\
& \quad=4 \pi \epsilon_{0} R \frac{1+R u^{-1}\left(\omega_{N}^{2}-\omega^{2}\right)^{1 / 2}}{1-R \omega^{2} u^{-1}\left(\omega_{N^{2}}-\omega^{2}\right)^{-1 / 2}} .
\end{aligned}
$$


Equations (6), (7), (9), (10), and (11) represent the main results of this paper, and a discussion of their significance follows here.

Equation (11) may also be used to calculate the impedance $Z_{\text {eff }}=2\left(i \omega C_{\text {eff }}\right)^{-1}$ of the antenna consisting of both spheres. The energy loss due to the radiation of electromagnetic waves has not as yet been included in the analysis; this will be done in a subsequent part of the present paper. The resistive part of the impedance $Z_{\text {eff }}$ represents only the energy loss due to the "radiation" of electrostatic waves.

It is convenient to express the impedance $Z_{\text {eff }}$ as a function of the parameters $Y=2\left(4 \pi \epsilon_{0} R \omega\right)^{-1}$ (twice the free space reactance of a sphere of radius $R$ ), $\delta=R u^{-1} \omega_{N}$ (the ratio of the radius $R$ to $\gamma^{1 / 2}$ times the Debye length), and $\psi=\omega / \omega_{N}$ (the ratio of the frequency to the electron plasma frequency). The result is

$$
Z_{\mathrm{eff}}=-i Y \frac{1-\delta \psi^{2}\left(1-\psi^{2}\right)^{-1 / 2}}{1+\delta\left(1-\psi^{2}\right)^{1 / 2}}
$$

It is clear from (12) that $Z_{\text {eff }}$ is purely imaginary for $\psi<1$ or $\omega<\omega_{N}$. The absence of a loss term is explained in this case by the absence of propagating electrostatic waves and the neglect of Landau damping. It may be seen from (12) that the impedance becomes infinitely high at the plasma frequency and vanishes at the frequency where $1-\delta \psi^{2}\left(1-\psi^{2}\right)^{-1 / 2}=0$. More will be said about the significance of this frequency later. At very low frequencies, $Z_{\text {eff }}$ $=-i Y(1+\delta)^{-1}$, and thus the impedance is smaller than the free space impedance by a factor $(1+\delta)^{-1}$. It is understandable that the effective capacity of the sphere is larger at low frequencies than the free space capacity because the alternating electric field does not extend to infinity, but is confined to within the plasma sheath, which in the case of large $\delta$ is much smaller than the radius. Figure 1 shows, for $\psi<1$, the ratio of the imaginary part of $Z_{\text {eff }}$ (the real part is zero for $\psi<1$ ) to the magnitude $Y$ of the free space reactance as a function of the ratio $\psi=\omega / \omega_{N}$ of the frequency to the electron plasma frequency for different values of the ratio $\delta$ of the radius to $\gamma^{1 / 2}$ Debye lengths. The curve for $\delta=\infty$ represents the well-known cold plasma approximation in which thermal motions are neglected and the plasma is treated as a dielectric with an effective dielectric constant $\epsilon_{0}\left(1-\omega_{N}{ }^{2} / \omega^{2}\right)$.

For $\psi>1$ it is convenient to write (12) in the form

$$
Z_{\mathrm{eff}}=\frac{Y \delta\left(\psi^{2}-1\right)^{-1 / 2}}{1+\delta^{2}\left(\psi^{2}-1\right)}-i \frac{Y\left(1+\delta^{2} \psi^{2}\right)}{1+\delta^{2}\left(\psi^{2}-1\right)} .
$$

Figure 1 also shows the real and the imaginary parts of $Z_{\text {eff }}$ for $\psi>1$ (in units of $Y$ ) as functions of $\psi$, for different values of $\delta$. The case of $\delta=\infty$ again represents the approximation in which thermal motions are neglected. The curves of figure 1 show the presence of a resistive component of the impedance for finite values of $\delta$. The resistive, ohmical component represents the energy loss caused by the radiation of electrostatic waves.

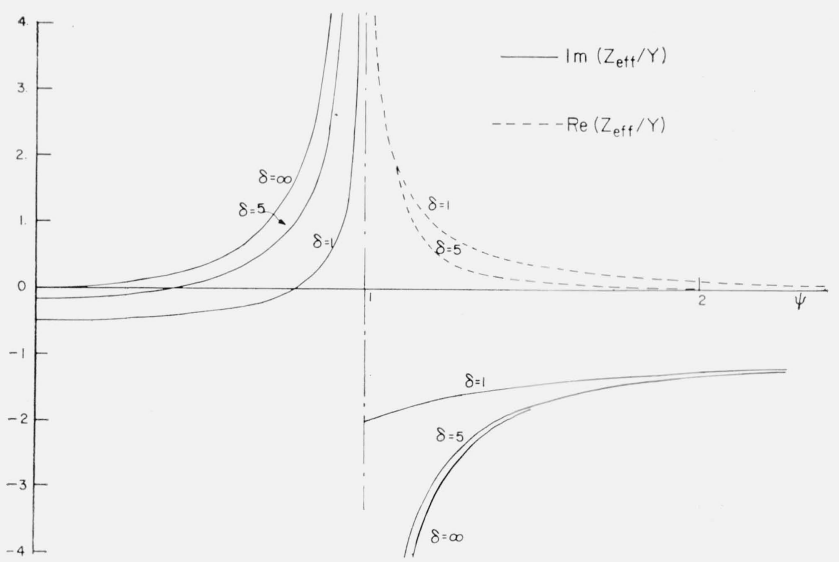

FIgURE 1. The ratios of the real (interrupted line) and imaginary (solid line) parts of the impedance to the magnitude of the free space reactance as functions of the ratio, $\Psi$, of the radio frequency to the electron plasma frequency.

\section{Radiation of Electromagnetic Waves by Two Spherical Conductors Excited in Antiphase}

The analysis of this paper can be extended to include radiation losses due to electromagnetic waves. The analysis of electromagnetic radiation by spheres oscillating in antiphase is, of course, somewhat artificial because in practice antennas resemble cylindrical conductors. The analysis of spheres oscillating in antiphase has, however, the advantage of simplicity and it illustrates the nature of the problem rather well. It would be relatively simple to extend the present analysis to cylindrical conductors, at least in an approximate manner. An exact formal solution for a slotted sphere antenna has been given by Wait [1964].

Since the distance $D$ between the two spheres is taken to be much smaller than the electromagnetic wavelength (and at the same time $R<\langle D$ ), the quasi-electrostatic field at a distance $r>D$ on the line connecting the spheres is $2 C_{2} D / r^{3}$, where $C_{2} / r$ is that part of the potential given by (7) which could be regarded as an approximation to the radiation field of a spherical radiator at distances much smaller than the electromagnetic wavelength. At distances much longer than a wavelength and in the plane that perpendicularly bisects the line connecting the two spheres, the magnitude $E$ of the radiation electric field of the dipole antenna is then given by

$$
E=C_{2} D k^{2} / r=\left(C_{2} D / r\right)\left[\left(\omega^{2}-\omega_{N^{2}}\right) / c^{2}\right] .
$$

The magnitude $H$ of the radiation magnetic field is given by

$$
H=\left(\epsilon_{0} C_{2} D / r\right)\left[\left(\omega^{2}-\omega_{N^{2}}\right)^{3 / 2} / c \omega\right]
$$

where $k$ is the wave number and $c$ is the velocity of electromagnetic waves in vacuum. Equations (14) and (15) were derived by fitting the quasi-electrostatic dipole field to the radiation field of a dipole 
[Stratton, 1941] in a medium with a dielectric constant $\epsilon_{0}\left(1-\omega_{N^{2}} / \omega^{2}\right)$. Equations (6), (7), and (9) can then be used to express $C_{2}$ in terms of $V(R)$, the alternating potential of one of the spheres, or in terms of the current supplied to the spherical conductors $I=i \omega C_{\text {eff }} V(R)$ where $C_{\text {eff }}$ is given by (11). The Poynting vector $\mathbf{E} \times \mathbf{H}$ may then be expressed in terms of $I$, and the total radiated power $P$ may be found by integration over a very large sphere. The result is

$$
P=\left(6 \pi \epsilon_{0}\right)^{-1} D^{2} \omega c^{-3}\left(\omega^{2}-\omega_{N^{2}}\right)^{1 / 2} I^{2}
$$

and therefore the resistive component of the antenna impedance (the radiation resistance) due to the radiation of electromagnetic waves is

$$
\rho_{e m}=\left(6 \pi \epsilon_{0}\right)^{-1} D^{2} \omega c^{-3}\left(\omega^{2}-\omega_{N}^{2}\right)^{1 / 2} .
$$

The resistive component due to electrostatic waves is given by (13) as

$$
\rho_{e s}=Y \frac{\delta\left(\psi^{2}-1\right)^{-1 / 2}}{1+\delta^{2}\left(\psi^{2}-1\right)} .
$$

Equations (17) and (18) show that $\rho_{e m}$ is zero at the electron plasma frequency and increases rapidly with frequency whereas $\rho_{e s}$ is infinitely high at the plasma frequency and decreases rapidly with frequency.

It is interesting to note that the expression (17) for $\rho_{e m}$ is the same as the expression for the radiation resistance of a short antenna of length $D$ (whose capacity is entirely at its extremities) situated in a medium with a dielectric constant $\epsilon_{0}\left(1-\omega_{N^{2}} / \omega^{2}\right)$. This is a significant result in view of the fact that in the calculation of the series reactive part of the antenna impedance the plasma cannot be replaced by a medium with a dielectric constant $\epsilon_{0}\left(1-\omega_{N^{2}} / \omega^{2}\right)$.

It is interesting to compare the ratio $\rho_{e m} / \rho_{e s}$ resulting from (17) and (18) with (73) of Wait for the corresponding ratio of the power $P_{p}$ radiated in the form of electrostatic waves to the power $P_{e}$ radiated in the form of electromagnetic waves by a slotted sphere antenna. Since Wait's equation (73) presupposes $k_{p} a>>1$ in Wait's notation, the corresponding assumption $k_{p} R>>1$ permits the neglect of unity in comparison with $\delta^{2}\left(\psi^{2}-1\right)$ in the denominator on the right of the present equation (18). Equations (17) and (18) of this paper then result in the equation:

$$
\frac{\rho_{e s}}{\rho_{e m}}=3 \frac{\omega_{N^{2}}}{\omega^{2}} \frac{u}{c} \frac{1}{D^{2} R^{2} k_{e}{ }^{2}}
$$

where Wait's definition of $k_{e}$ is used. The factor $2 a^{-4}$ in Wait's equation (73) has been replaced by a factor $3 D^{-2} R^{-2}$ in (19) but otherwise the two equations are identical. The similarity of the present result to that of Wait has thus been demonstrated for the special case $k_{p} R>>1$.

\section{Application to Resonance Probes}

The results of the previous section may be used to draw some tentative but very important conclusions about the behavior of resonance probes [Miyazaki et al., 1960]. In such probes the change, caused by the application of a radiofrequency voltage in the direct current collected by a Langmuir probe, is measured as a function of the radiofrequency. It has usually been accepted [Miyazaki et al., 1960] as an experimental fact that the change in the collected current shows a resonant increase at the plasma frequency.

In this section the point of view is taken that the change in the collected direct current is due to rectification caused by the nonlinear characteristic of the Langmuir probe. The amplitude of the radiofrequency variations in the collected current will be proportional to the fluctuations $n(R)$ in the number density (in a more accurate treatment the fluctuation in temperature would also have to be taken into account but these would in any case be proportional to the fluctuations in number density) and therefore (10) should roughly describe the characteristics of a resonance probe. Using the parameters $\delta$ and $\psi$, (10) may be written in the form

$$
n(R)=V(R) \frac{\epsilon_{0} \omega_{N}^{2}}{e u^{2}}\left(1-\frac{\delta \psi^{2}}{\left(1-\psi^{2}\right)^{1 / 2}}\right)^{-1} .
$$

Equation (20) shows that $n(R)$ becomes infinitely large at the frequency for which

$$
\psi=\left\{2^{-1} \delta^{-2}\left[-1+\left(1+4 \delta^{2}\right)^{1 / 2}\right]\right\}^{1 / 2} .
$$

This shows that resonance occurs near the electron plasma frequency $(\psi \sim 1)$ only when $\delta<<1$; for large values of $\delta$ the resonance occurs well below the plasma frequency. Figure 2 shows $\left(e u^{2} / \omega_{N^{2}} \epsilon_{0}\right) \mid n(R) /$ $V(R) \mid$ as a function of $\psi$ for two values of $\delta$ and illustrates that resonance occurs well below the

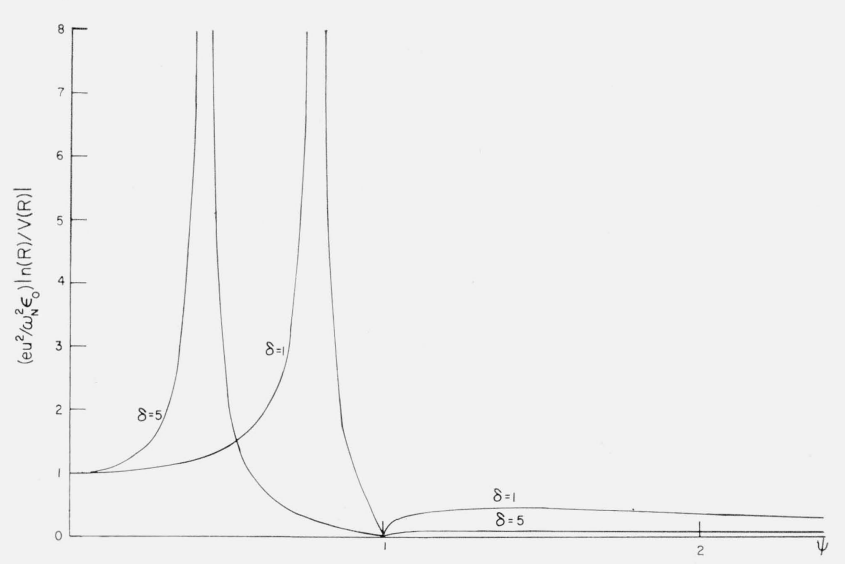

Figure 2. The dimensionless quantity $\left(\mathrm{eu}^{2} / \omega_{\mathrm{N}}{ }^{2} \epsilon_{\mathrm{o}}\right) \mid \mathrm{n}(\mathrm{R}) /$ $\mathrm{V}(\mathrm{R}) \mid$, which is proportional to the number density fluctuations, $\mathrm{n}(\mathrm{R})$, produced by an rf voltage of given amplitude $\mathrm{V}(\mathrm{R})$, as a function of the ratio, $\Psi$, of the radio frequency to the electron plasma frequency. 
plasma frequency when the probe radius is much larger than the Debye length. The resonant frequency given by (21) is the same as the previously discussed frequency where the impedance of the probe vanishes.

The present analysis clearly leads to the conclusion that the resonant response of the probe does not occur at the plasma frequency, as laboratory and space experiments are claimed to show, but always below the plasma frequency. Large errors could thus occur in rocket investigations of electron concentration in which the resonant frequency was identified with the plasma frequency, especially with probes whose radii were much larger than the Debye length. Fortunately a relatively small sphere $(R=$ $1 \mathrm{~cm}$ ) was used in the ionospheric experiments [Aono et al., 1962] reported so far.

A discussion of the physical nature of this resonance sheds some light on the reasons for the occurrence of the resonance well below the plasma frequency. It is clear from (7) that the electric field of the probe consists of two parts. One is simply a quasielectrostatic field, which at short distances is the approximate form of the radiation field for frequencies above the plasma frequency whereas the other is the field associated with an electrostatic wave which is evanescent at frequencies below the plasma frequency.

At very low frequencies the quasi-electrostatic field becomes very small compared to the field of the evanescent electrostatic wave. This means that the alternating charge on the conducting sphere is almost perfectly shielded by a suitable (continuous) modification of the sheath. As the frequency is increased, the shielding becomes less perfect and a potential $C_{2} / r$ appears outside the sheath. The present theory shows that the outside field opposes in phase the field within the sheath. With increasing frequency a situation is reached eventually where the potential drop outside the sheath just balances the potential drop inside so that no exciting voltage is required on the conductor and resonant oscillations occur.

This explanation of the resonance is strongly supported by the full agreement, in one special case, of the present approximate calculation with Landau's [1946] more accurate analysis utilizing the collisionless Boltzmann equation. Landau considered the penetration of an alternating electric field, that is normal to a plane boundary, from empty space on the one side of the boundary into a uniform plasma on the other side. He showed that at frequencies slightly below the plasma frequency the field asymptotically approaches a value which is equal to $\left(1-\omega_{N^{2}} / \omega^{2}\right)^{-1}$ times its value at the boundary. The field deep inside the plasma is thus out of phase with the field at the boundary. Equations (6), (7), and (9) of the present paper lead to the same asymptotic value if $R$ and $r-R$ both approach infinity and at the same time satisfy the inequality $R>r-R$. Landau also showed that the asymptotic value is approached according to a simple exponential law expressed by his equation (45). Essentially the same exponential law with the same exponent $(-\alpha r$ in equation 6) results from (6), (7), and (9) of this paper.

The above conclusions about the behavior of the resonance probe described by Miyazaki et al. [1960] are in qualitative agreement with most of the conclusions of Harp [1963] who used an approximate modification of results, obtained with the aid of the collisionless Boltzmann equation by Pavkovich [1964], to spherical geometry. The most important difference between the present treatment and that of Harp [1963] and Pavkovich [1964] is the neglect of Landau damping by the present treatment; this damping appears to reduce the resonance effect drastically for probes whose radius is smaller than about twice the Debye length [Harp, 1963].

\section{Conclusions}

The simple analysis of this paper leads to certain interesting and significant results about the behavior of antennas in plasmas and about the interpretation of observations with the aid of resonance probes in plasmas. It is shown that resonance does not take place at the plasma frequency and that previous measurements made by the resonant probe method may have to be reinterpreted. In principle both the electron concentration and the electron temperature could be determined by simultaneous measurements of the resonant frequencies of two resonance probes of different size. Alternatively the impedance of a single probe could be measured at two different frequencies. A theory that is more accurate than that of the present paper or than other presently available theories, would, however, be required for the accurate interpretation of such measurements.

The benefit of discussions with Drs. J.'E.Midgley, H. B. Liemohn, and W. B. Hanson and of correspondence with Professor Gordon S. Kino is acknowledged. Thanks are due to Miss J. Ligon for assistance with the computations. This research was supported by the National Aeronautics and Space Administration under grant NsG-269--62.

\section{References}

Aono, Y., K. Hirao, and S. Miyazaki (1962), Profile of charged particle density in the ionosphere observed with rockets, Space Research III, pp. 221-227 (North-Holland Publishing Co., Amsterdam).

Chen, Kun-Mu (1963), Interaction of a radiating source with a plasma effect of an electroacoustic wave, International Symposium on Space Telecommunications, July 9-11, 1963 in Boulder, Colo., Program and Digest, 252-257 (published by IEEE, New York, N.Y.).

Cohen, M. H. (1961), Radiation in a plasma. I. Cerenkov effect, Phys. Rev. 123, 711-721.

Cohen, M. H. (1962a), Radiation in a plasma. II. Equivalent sources, Phys. Rev. 126, 389-397.

Cohen, M. H. (1962b), Radiation in a Plasma. III. Metal boundaries, Phys. Rev. 126, 398-404.

Fejer, J. A. (1964), Scattering of electromagnetic waves by a plasma cylinder, Phys. Fluids $\boldsymbol{7}$, No. 3, 439-445.

Gould, R. W. (1959), Experiments on plasma oscillations Proceedings of the Conference on Plasma Oscillations. pp. 167-204 (Linde Company, Indianapolis, Ind.). 
Harp, R. S. (November 1963), A theory of the resonance probe, Microwave Laboratory Report No. 1117, Stanford University.

Hessel, A. and J. Shmoys (1962), Excitation of plasma waves in a homogeneous isotropic plasma by a dipole, Proc. Symposium on Electromagnetic and Fluid Dynamics of Gaseous Plasma, 173-184 (Polytechnic Press, Brooklyn, N.Y.).

Landau, L. (1946), On the vibrations of the electronic plasma, J. Phys. U.S.S.R. 10, 25-34.

Miyazaki, S., K. Hirao, Y. Aono, K. Takayama, H. Ikegami, and T. Ichimiya (1960), Resonance probe a new probe method for measuring electron density and electron temperature in the ionosphere, Rep. Ionos. Space Res. Japan 14, 148-159.

Nickel, J. C., J. V. Parker, and R. W. Gould (1963), Resonance oscillations in a hot nonuniform plasma column, Phys. Rev. Letters 11, 183-185.
Pavkovich, J. M. (January, 1964), Numerical calculations related to the RF properties of the plasma sheath, Microwave Laboratory Report No. 1093, Stanford University.

Spitzer, L., Jr. (1962), Physies of Fully Ionized Gases, p. 17 (Interscience Publishers, New York and London).

Stratton, J. A. (1941), Electromagnetic Theory (McGrawHill Book Co. Inc., New York, N.Y.).

Tonks, L. and I. Langmuir (Feb. 1929), Oscillations in ionized gases, Phys. Rev. 33, 195-210.

Wait, J. R. (1964), Theory of a slotted-sphere antenna immersed in a compressible plasma. Part I. Radio Sci. J. Res. NBS/USNC-URSI 68D, No. 10, pp. 1127-1136.

Whale, H. A. (1963), The excitation of electroacoustic waves in the ionosphere, J. Geophys. Res. 68, 415-422.

(Paper 68D11-417) 